International Mathematical Forum, 2, 2007, no. 66, 3285 - 3291

\title{
On the Regularity of Two Families of Finitely Presented $p$-Groups
}

\author{
M. Hashemi \\ Mathematics Department, Faculty of Science \\ Guilan University, P. O. Box 451 Rasht, Iran \\ m_hashemi@guilan.ac.ir
}

\begin{abstract}
Since 1934, the regular $p$-groups have been studied by Hall. A regular $p$-group is power closed, exponent closed, strongly semi- $p$-abelian and an exact power margin group. In this paper we study the regularity of two families of finitely presented $p$-groups where $p \geq 3$.
\end{abstract}

Mathematics Subject Classification: 20D15, 20F99

Keywords: Groups, p-group, regularity

\section{Introduction}

Many authors have been studied the power structure, for example one may see $[2,3,6]$. In particular, F. L. Kluempen [4] investigated power structure for two generator 2-groups of nilpotency class two.mmnot $\mid n$

Let $G$ be a finite $p$-group. We define a mapping $\Pi: G \longrightarrow G$ by $\Pi_{i}(g)=$ $g^{p^{i}}$ for all $g \in G$. Denoted $\bigvee_{i}(G)=i m g\left(\Pi_{i}\right)=\left\{g^{p^{i}} \mid g \in G\right\}$ and $\Lambda_{i}(G)=$ $\operatorname{ker}\left(\Pi_{i}\right)=\left\{g \mid g^{p^{i}}=1\right\}$. Set $\Delta_{i}(G)=\left\langle\bigvee_{i}(G)\right\rangle$ and $\Omega_{i}(G)=\left\langle\bigwedge_{i}(G)\right\rangle$. Using by this notation, we make the following definitions.

Definition 1.1. Let $G$ be a finite $p$-group. We say that $G$ is power closed if $\bigvee_{i}(G)=\Delta_{i}(G)$ for all $i \in N$, and $G$ is exponent closed if $\Lambda_{i}(G)=\Omega_{i}(G)$ for all $i \in N$.

Given a word $f\left(x_{1}, x_{2}, \ldots, x_{m}\right)$ in the variables $x_{1}, x_{2}, \ldots, x_{m}$, the marginal subgroup of a group $G$ is the set $\left\{a \in G \mid f\left(g_{1}, \ldots, a g_{i}, \ldots, g_{m}\right)=f\left(g_{1}, \ldots, g_{m}\right)\right.$ for $1 \leq i \leq m$ and $\left.g_{1}, \ldots, g_{m} \in G\right\}$. For a $p$-group $G$, the $p^{i}$-power margin is $M_{i}(G)=\left\{a \in G \mid(a g)^{p^{i}}=g^{p^{i}}, \forall g \in G\right\}$. Now we give the following definition. 
Definition 1.2.[3]. A $p$-group $G$ is said to be exact power margin if $M_{i}(G)=$ $\bigwedge_{i}(G)$ for all $i \in N$. Also we say that $G$ is semi- $p^{i}$-abelian, provided that $(x y)^{p^{i}}=1$ if and only if $x^{p^{i}} y^{p^{i}}=1$ for all $x, y \in G$; and $G$ is strongly semi- $p$ abelian, provided $G$ is semi- $p^{i}$-abelian for all $i \in N$.

We consider two families of finitely presented $p$-groups as:

$$
\begin{aligned}
& G_{n}=\left\langle a, b \mid a^{n}=b^{n}=1,[a, b]^{a}=[a, b],[a, b]^{b}=[a, b]\right\rangle, \quad n \geq 1, \\
& H_{m}=\left\langle x, y \mid x^{m^{2}}=y^{m}=1, y^{-1} x y=x^{1+m}\right\rangle, \quad m \geq 2 .
\end{aligned}
$$

If $n=2^{t}$ (or $m=2^{t}$ ) the power structure of $G_{n}$ (or $H_{m}$ ) was investigated in [4]. In this paper we will study in the otherwise.

In section 2 we study the group $G_{n}$ and show that for an integer $t \geq 1$ and for a prime $p \geq 3$, let $n=p^{t}$ then $G_{n}$ is regular. Section 3 is devoted to study of the groups $H_{n}$

\section{The regularity of $G_{n}$}

First we state a lemma without proof that establishes some properties of $G_{n}$.

Lemma 2.1. Let $k$ be an integer and $x, y, z \in G=G_{n}$. Then

(i) $G^{\prime} \subseteq Z(G)$.

(ii) $[x y, z]=[x, y][y, z]$ and $[x, y z]=[x, y][x, z]$.

(iii) $\left[x^{k}, y\right]=\left[x, y^{k}\right]=[x, y]^{k}$.

(iv) $(x y)^{k}=x^{k} y^{k}[y, x]^{k(k-1) / 2}$.

Also, We recall the following lemma of [1].

Lemma 2.2. Let $G=G_{n}$ then $\left|G_{n}\right|=n^{3},|Z(G)|=n$ and $Z(G)=G^{\prime}=$ $\left\langle x \mid x^{n}=1\right\rangle$.

We now show that every element in the $G_{n}$, where $n \in N$, has standard form:

Lemma 2.2. For every element of the group $G=G_{n}$ can be written uniquely in the form $a^{i} b^{j}[b, a]^{k}$ where $0 \leq i, s, k \leq n-1$. 
Proof. Since $[a, b]^{a}=[a, b],[a, b]^{b}=[a, b]$, then $[a, b] \in Z(G)$ also

$$
\begin{aligned}
{\left[a, b^{-1}\right] } & =\left([a, b]^{b^{-1}}\right)^{-1} \in Z(G), \\
{\left[a^{-1}, b\right] } & =\left([a, b]^{a^{-1}}\right)^{-1}=[a, b]^{-1} \in Z(G) .
\end{aligned}
$$

Also, for every $x=x_{1}^{s_{1}} x_{2}^{s_{2}} \ldots x_{k}^{s_{k}}$ in $G_{n}$ where $x_{i} \in\{a, b\}$ and $s_{1}, s_{2}, \ldots, s_{k}$ are integers, and using the relations $b^{j} a^{i}=a^{i} b^{j}\left[b^{j}, a^{i}\right]$, we may easily prove that every element of $G$ is in the form $a^{i} b^{j} g$ where $0 \leq i<m-1,0 \leq j \leq n-1$ and $g \in G^{\prime}$ (by induction method on the length of the word $x$.) Suppose $x=a^{i} b^{j} g=e$ then $a^{i} b^{j} \in Z(G)$ and $\left[a, b^{j}\right]=[a, b]^{j}=1$ so $n \mid j$. Similarly $n \mid i$, that is $i=j=0$ and $g=e$. The result is now immediate.

For an integer $t \geq 1$ and for a prime $p \geq 3$, let $n=p^{t}$ and $x=a^{r_{1}} b^{s_{1}}[b, a]^{t_{1}}, y=$ $a^{r_{2}} b^{s_{2}}[b, a]^{t_{2}} \in G$ then by $2.1,2.2$ and 2.3 we get

$$
\begin{cases}x y & =a^{r_{1}} b^{s_{1}}[b, a]^{t_{1}} a^{r_{2}} b^{s_{2}}[b, a]^{t_{2}}=a^{r_{1}+r_{2}} b^{s_{1}+s_{2}}[b, a]^{s_{1} r_{2}+t_{1}+t_{2}} \\ x^{p^{i}} & =a^{p^{i} r_{1}} b^{p^{i} s_{1}}\left[b^{s_{1}}, a^{r_{1}}\right]^{p^{i}\left(p^{i}-1\right) / 2}[b, a]^{p^{i} t_{1}}=a^{p^{i} r_{1}} b^{p^{i} s_{1}}[b, a]^{p^{i} t_{1}+r_{1} s_{1} p^{i}\left(p^{i}-1\right) / 2} \\ x^{p^{i}} y^{p^{i}} & =a^{p^{i}\left(r_{1}+r_{2}\right)} b^{p^{i}\left(s_{1}+s_{2}\right)}\left[b^{p^{i} s_{1}}, a^{p^{p_{1}} r_{1}}\right][b, a]^{p^{i}\left(t_{1}+t_{2}\right)+\left(r_{1} s_{1}+r_{2} s_{2}\right) p^{i}\left(p^{i}-1\right) / 2} \\ & =a^{p^{i}\left(r_{1}+r_{2}\right)} b^{p^{i}\left(s_{1}+s_{2}\right)}[b, a]^{p^{i}\left(t_{1}+t_{2}\right)+p^{2 i} s_{1} r_{2}+\left(r_{1} s_{1}+r_{2} s_{2}\right) p^{i}\left(p^{i}-1\right) / 2} \\ (x y)^{p^{i}} & =a^{p^{i}\left(r_{1}+r_{2}\right)} b^{p^{i}\left(s_{1}+s_{2}\right)}[b, a]^{\left(s_{1}+s_{2}\right)\left(r_{1}+r_{2}\right) p^{i}\left(p^{i}-1\right) / 2}[b, a]^{p^{i}\left(s_{1} r_{2}+t_{1}+t_{2}\right)} \\ & =a^{p^{i}\left(r_{1}+r_{2}\right)} b^{p^{i}\left(s_{1}+s_{2}\right)}[b, a]^{p^{i}\left(s_{1} r_{2}+t_{1}+t_{2}\right)+\left(s_{1}+s_{2}\right)\left(r_{1}+r_{2}\right) p^{i}\left(p^{i}-1\right) / 2}\end{cases}
$$

The following proposition is the main result of this section

Proposition 2.3. For every prime $p \geq 3$ and every integer $k \geq 1$, let $n=p^{k}$ then $G=G_{n}$ is regular.

Proof. By definition of regularity, we should prove that $G$ is power closed, exponent closed, exact power margin group and strongly semi-p-abelian

For every $i \geq 1$ and for every $g_{1}^{p^{i}}, g_{2}^{p^{i}} \in \bigvee_{i}(G)$ we get $g_{1}^{p^{i}} g_{2}^{p^{i}}=\left(g_{1} g_{2}\left[g_{1}, g_{2}\right]^{\left(p^{i}-1\right) / 2}\right)^{p^{i}}\left(\right.$ by 2.1.4) so $\bigvee_{i}(G)$ is subgroup of $G$ and $\bigvee_{i}(G)=$ $\Delta_{i}(G)$.

For exponent closed, suppose $g_{1}, g_{2} \in \Lambda_{i}(G)$ thus

$$
\left(g_{1} g_{2}^{-1}\right)^{p^{i}}=g_{1}^{p^{i}} g_{2}^{-p^{i}}\left[g_{2}^{-1}, g_{1}\right]^{p^{i}\left(p^{i}-1\right) / 2}=\left[g_{2}^{-1}, g_{1}^{p^{i}}\right]^{\left(p^{i}-1\right) / 2}=1 .
$$

Consequently $\Lambda_{i}(G)$ is subgroup of $G$ and $\Lambda_{i}(G)=\Omega_{i}(G)$ for all $i \in N$. Clearly $M_{i}(G)=\left\{z \in G \mid(z g)^{p^{i}}=g^{p^{i}}, \forall g \in G\right\}$ is subset of $\Lambda_{i}(G)$. Let $x \in \Lambda_{i}(G)$ then for every $g \in G$, we conclude that;

$$
(x g)^{p^{i}}=x^{p^{i}} g^{p^{i}}[g, x]^{p^{i}\left(p^{i}-1\right) / 2}=g^{p^{i}}\left[g, x^{p^{i}}\right]^{\left(p^{i}-1\right) / 2}=g^{p^{i}} .
$$


Therefore $x \in M_{i}(G)$ and $G$ is an exact power margin group.

Lastly, we prove that $G$ is strongly semi- $p$-abelian. Let $x=a^{r_{1}} b^{s_{1}}[b, a]^{t_{1}}, y=$ $a^{r_{2}} b^{s_{2}}[b, a]^{t_{2}} \in G$. Then by (1) and the Lemma 2.2, we get $(x y)^{p^{i}}=1$ if and only if all of the following equations

$$
\left\{\begin{array}{l}
p^{i}\left(r_{1}+r_{2}\right) \equiv 0\left(\bmod p^{k}\right) \\
p^{i}\left(s_{1}+s_{2}\right) \equiv 0\left(\bmod p^{k}\right) \\
p^{i}\left(t_{1}+t_{2}+r_{2} s_{1}\right) \equiv 0\left(\bmod p^{k}\right)
\end{array}\right.
$$

hold (for, $p^{i}\left(r_{1}+r_{2}\right) \equiv 0\left(\bmod p^{k}\right)$ and $[b, a]^{p^{k}} \equiv 0\left(\bmod p^{k}\right)$.) Similarly, $x^{p^{i}} y^{p^{i}}=$ 1 if and only if

$$
\left\{\begin{array}{l}
p^{i}\left(r_{1}+r_{2}\right) \equiv 0\left(\bmod p^{k}\right) \\
p^{i}\left(s_{1}+s_{2}\right) \equiv 0\left(\bmod p^{k}\right) \\
p^{i}\left(t_{1}+t_{2}\right)+p^{2 i} s_{1} r_{2}+\left(r_{1} s_{1}+r_{2} s_{2}\right) p^{i}\left(p^{i}-1\right) / 2 \equiv 0\left(\bmod p^{k}\right)
\end{array}\right.
$$

Since, $p^{i} r_{1} \equiv-p^{i} r_{2}\left(\bmod p^{k}\right)$ and $p^{i} s_{1} \equiv-p^{i} s_{2}\left(\bmod p^{k}\right)$ then

$p^{2 i} s_{1} r_{2}+\left(r_{1} s_{1}+r_{2} s_{2}\right) p^{i}\left(p^{i}-1\right) / 2 \equiv p^{2 i} s_{1} r_{2}-\left(2 r_{2} s_{1}\right) p^{i}\left(p^{i}-1\right) / 2 \equiv p^{i} s_{1} r_{2}\left(\bmod p^{k}\right)$

Therefore, the equations (2) and (3) are equivalent and this yields, $G$ is strongly semi- $p$-abelian.

\section{The regularity of $H_{m}$}

Consider $G=H_{m}=\left\langle x, y \mid x^{m^{2}}=y^{m}=1, y^{-1} x y=x^{1+m}\right\rangle, \quad m \geq 2$. In this section we study the regularity of $H_{n}$. First we need the following lemma.

Proposition 3.1. Let $G=H_{m}$. Then $Z(G)=G^{\prime} \simeq\left\langle z \mid z^{m}=1\right\rangle$.

Proof. We first prove that $G^{\prime} \subseteq Z(G)$. By the relations of $G$ we get $[x, y]=$ $x^{-1} x^{y}=x^{-1} x^{1+m}=x^{m}$. Then

$$
\left\{\begin{aligned}
{[[x, y], y] } & =y^{-1} x^{-1} y x y^{-1} x^{-1} y^{-1} x y^{2}=\left(x^{-1}\right)^{y} x\left(x^{-1}\right)^{y} x^{y^{2}} \\
& =x^{-m} x^{-1-m} x^{(1+m)^{2}}=x^{-2 m-1} x^{1+2 m+m^{2}} \\
& =x^{m^{2}}=1
\end{aligned}\right.
$$

And also the relation $[[x, y], x]=1$ holds, so $G^{\prime} \subseteq Z(G)$ and $[x, y]^{m}=1$.

It is sufficient to show that $Z(G) \subseteq G^{\prime}$. For every $U=u_{1}^{s_{1}} u_{2}^{s_{2}} \ldots u_{k}^{s_{k}}$ in $G$, where $u_{i} \in\{x, y\}$ and $s_{1}, s_{2}, \ldots, s_{k}$ are integers, using the relation $y^{-1} x y=$ 
$x^{1+m}$, we may easily prove that $U$ is in the form $y^{r} x^{s}$ where $0 \leq r<m, 0 \leq$ $s \leq m^{2}$. Suppose $y^{r} x^{s} \in Z(G)$ so $y^{r} x=x y^{r}$ and $y x^{s}=x^{s} y$. Then

$$
\begin{gathered}
1=\left[x, y^{r}\right]=x^{-1} x^{(1+m)^{r}}=x^{-1} x^{(1+r n)}=x^{r m} \\
1=\left[x^{s}, y\right]=x^{-s}\left(x^{s}\right)^{y}=x^{-s} x^{(1+m) s}=x^{m s} .
\end{gathered}
$$

This shows that $m \mid r$ and $m \mid s$, and then $y^{r} x^{s}=\left(x^{m}\right)^{t}=[x, y]^{t} \in G^{\prime}$. Therefore $Z(G)=G^{\prime}$.

Corollary 3.2. Let $G=H_{m}$. Then $|G|=m^{3}$.

Proof. By the above calculations, for every element of the group $G=H_{m}$ can be written in the form $y^{r} x^{s}$ where $0 \leq r \leq m-1$ and $0 \leq s \leq m^{2}-1$. Now, let $y^{r} x^{s}=1$ then $1=\left[y^{r} x^{s}, y\right]=[x, y]^{s}$. Therefore $m\left|s, m^{2}\right| r$ and uniqueness of the presentation follows. This yield that $|G|=m^{3}$.

For an integer $k \geq 1$ and for a prime $p \geq 3$, let $m=p^{k}$ and $u=y^{r_{1}} x^{s_{1}}, v=$ $y^{r_{2}} x^{s_{2}} \in G$. When we are trying to prove that the regularity of $H_{m}$ we need to concentrate on the terms $u v, u^{p^{i}}$ and $(u v)^{p^{i}}$. By 2.1, 3.1 and 3.2 we get

$$
\begin{aligned}
& u v=y^{r_{1}} x^{s_{1}} y^{r_{2}} x^{s_{2}}=y^{r_{1}+r_{2}} x^{s_{1}+s_{2}}[b, a]^{s_{1} r_{2}}=y^{r_{1}+r_{2}} x^{s_{1}+s_{2}+m s_{1} r_{2}} \\
& u^{p^{i}}=y^{p^{i} r_{1}} x^{p^{i} s_{1}}\left[b^{s_{1}}, a^{r_{1}}\right]^{p^{i}\left(p^{i}-1\right) / 2}=y^{p^{i} r_{1}} x^{p^{i} s_{1}+m r_{1} s_{1} p^{i}\left(p^{i}-1\right) / 2} \\
& u^{p^{i}} v^{p^{i}}=y^{p^{i}\left(r_{1}+r_{2}\right)} x^{p^{i}\left(s_{1}+s_{2}\right)+m\left(r_{1} s_{1}+r_{2} s_{2}\right) p^{i}\left(p^{i}-1\right) / 2}[x, y]^{p^{i} r_{2}\left(p^{i} s_{1}+m r_{1} s_{1} p^{i}\left(p^{i}-1\right) / 2\right.} \\
& =y^{p^{i}\left(r_{1}+r_{2}\right)} x^{p^{i}\left(s_{1}+s_{2}\right)+m p^{2 i} s_{1} r_{2}+m\left(r_{1} s_{1}+r_{2} s_{2}\right) p^{i}\left(p^{i}-1\right) / 2} \\
& (u v)^{p^{i}}=\left(y^{r_{1}+r_{2}} x^{s_{1}+s_{2}+m s_{1} r_{2}}\right)^{p^{i}}=y^{p^{i}\left(r_{1}+r_{2}\right)} x^{p^{i}\left(s_{1}+s_{2}+m s_{1} r_{2}\right)} \\
& \times[x, y]^{p^{i}\left(r_{1}+r_{2}\right)\left(s_{1}+s_{2}+m s_{1} r_{2}\right)\left(p^{i}-1\right) / 2} \\
& =y^{p^{i}\left(r_{1}+r_{2}\right)} x^{p^{i}\left(s_{1}+s_{2}+m s_{1} r_{2}\right)+\left(r_{1}+s_{1}\right)\left(r_{2}+s_{2}\right) m p^{i}\left(p^{i}-1\right) / 2}
\end{aligned}
$$

We can now proceed to the main result of this section.

Proposition 3.3. For every prime $p \geq 3$ and every integer $k \geq 1$, let $m=p^{k}$ then $G=H_{m}$ is regular. 
Proof. In a similar way as for the Proposition 2.3, we can prove that $G$ is power closed, exponent closed and exact power margin group. Now we prove that $G$ is strongly semi-p-abelian. Let $u=y^{r_{1}} x^{s_{1}}, v=y^{r_{2}} x^{s_{2}} \in G$ then by (4) and the proof of corollary 3.2, we get $(u v)^{p^{i}}=1$ if and only if all of the following equations

$$
\left\{\begin{array}{l}
p^{i}\left(r_{1}+r_{2}\right) \equiv 0(\bmod m) \\
p^{i}\left(s_{1}+s_{2}+m s_{1} r_{2}\right)+\left(r_{1}+r_{2}\right)\left(s_{1}+s_{2}\right) m p^{i}\left(p^{i}-1\right) / 2 \equiv 0\left(\bmod m^{2}\right)
\end{array}\right.
$$

hold. Since $\left(r_{1}+r_{2}\right)\left(s_{1}+s_{2}\right) m p^{i}\left(p^{i}-1\right) / 2 \equiv 0\left(\bmod m^{2}\right)$ then it is equivalent with

$$
\left\{\begin{array}{l}
p^{i}\left(r_{1}+r_{2}\right) \equiv 0(\bmod m) \\
p^{i}\left(s_{1}+s_{2}+m s_{1} r_{2}\right) \equiv 0\left(\bmod m^{2}\right) \\
s_{1}+s_{2} \equiv 0(\bmod m)
\end{array}\right.
$$

Also $u^{p^{i}} v^{p^{i}}=1$ if and only if

$$
\left\{\begin{array}{l}
p^{i}\left(r_{1}+r_{2}\right) \equiv 0(\bmod m) \\
p^{i}\left(s_{1}+s_{2}\right)+m p^{2 i} s_{1} r_{2}+\left(r_{1} s_{1}+r_{2} s_{2}\right) m p^{i}\left(p^{i}-1\right) / 2 \equiv 0\left(\bmod m^{2}\right) \\
s_{1}+s_{2} \equiv 0(\bmod m)
\end{array}\right.
$$

Since, $p^{i} r_{1} \equiv-p^{i} r_{2}(\bmod m)$ and $p^{i} s_{2} \equiv-p^{i} s_{1}(\bmod m)$ then

$$
\begin{gathered}
m p^{2 i} s_{1} r_{2}+m\left(r_{1} s_{1}+r_{2} s_{2}\right) p^{i}\left(p^{i}-1\right) / 2 \equiv m p^{2 i} s_{1} r_{2}-m\left(2 r_{2} s_{1}\right) p^{i}\left(p^{i}-1\right) / 2 \\
\equiv m p^{i} s_{1} r_{2}\left(\bmod m^{2}\right)
\end{gathered}
$$

Therefore, the equations (5) and (6) are equivalent and this yields, $G$ is strongly semi- $p$-abelian.

\section{References}

[1] H. Doostie, and M. Hashemi, Fibonacci Lengths involvig the Wall number $k(n)$, J. Appl.Math. Computing Preprint.

[2] P. Hall, Acontribution to the theory of groups of prime-power order, Proc. Londan Math. Soc. 36 (1934) 29-95.

[3] L. C. Kappe, On power margins, J. Algebra 122 (1989) 337-344. 
[4] F. L. Kluempen, The power structure of 2-generator 2-groups of class two, Algebra Colloquium 9:3 (2002) 287-302.

[5] M. Xu, On semi-p-abelian p-groups and their power structure, Acta. Math. Sinica 23 (1980) 78-87 (in Chinese).

[6] M. Xu, The power structure of finite p-groups, Bull. Austral. Math. Soc. 36 (1987) 1-10.

Received: June 24, 2007 